\title{
Design of Multi-voice Electronic Piano by Chip Microcomputer
}

\author{
Wei Li \\ School of China West Normal University, Nanchong 637000, China \\ dxlw@qq.com
}

Keywords: timbre, electronic panio, filtering network, chip microcomputer, D/A.

\begin{abstract}
The concept of timbre and the critical factors which affect timbre is described. Traditionally, we simulate the timbre wave by using filtering network which magnify or attenuate some harmonic wave content. It is simulated the wave by using a chip microcomputer which is controlled by software. The main advantages of the new strategy is regularization and simplification of the design of circuit. It gave the detailed circuit and the of software.
\end{abstract}

\section{Introduction}

The single chip microcomputer (SCM) is widely used in many fields due to its small volume, fast speed, low power consumption, reliable performance, convenient use, low price and other characteristics. In this paper, the electronic organ which simulates multiple timbres with SCM is introduced. SCM used in the examples of this paper are common AT89C52. There is an additional DAC0832 SCM for loudspeaker.

\section{Musical knowledge required by making the electronic organ}

Sound is produced by the vibration of objects and musical sound is the result of regular vibration. The musical sound has some basic characteristics, such as key and timbre.

The key is decided by the vibration frequency. For example, the frequency of the most familiar keys, 1, 2, 3, 4, 5, 6, 7, 1, is the periodic signal of 261.63, 293.66, 329.63, 349.23, 392, 440, 493.9, 523.26 respectively (this is the value confirmed according to the piano intonation). Either guitar or Erhu, the frequency of do key is the same; otherwise, the key is modified and intonation should be corrected. The adjustment on the tightness of strings to make the intonation reach the standard is essentially to adjust the frequency of musical key.

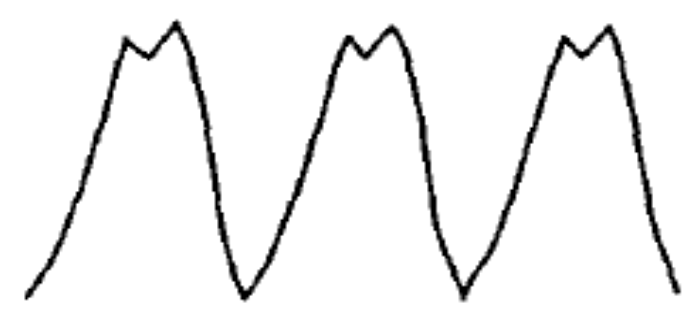

Fig. 1 The flute music wave

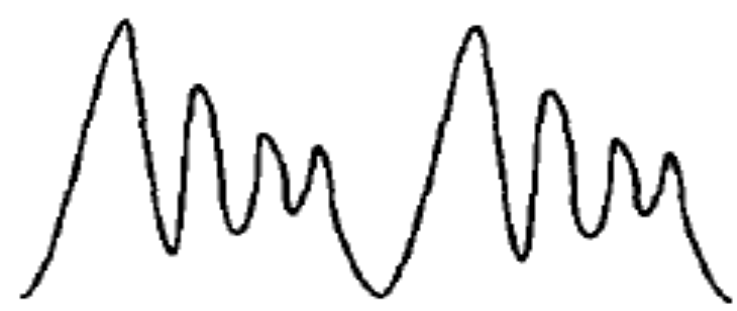

Fig. 2 Saxophone music wave 
Timbre is another important feature of musical sound. For example, The Butterfly Lovers sounds different by saxophone and flute and people can distinguish them immediately. Why? As shown in figure 1 and 2, their vibration waveforms are different. If the waveforms of the signal of two musical sounds are the same, their timbres are also the same. This is very important.

\section{Theoretical realization plan}

It is known from the above-mentioned analysis that the key and timbre of musical sound can be determined by controlling its frequency (period) and waveform.

According to the disciplinary knowledge, a non-sinusoidal wave periodic signal can be decomposed into fundamental wave and a series of harmonic component. The formula is as follows:

$F(\mathrm{wt})=A_{0}+A_{1} \sin \left(\mathrm{wt}+\varphi_{1}\right)+A_{2} \sin \left(2 \mathrm{wt}+\varphi_{2}\right)+A_{3} \sin \left(3 \mathrm{wt}+\varphi_{3}\right)+\ldots+A_{\mathrm{K}}\left(\mathrm{kwt}+\varphi_{\mathrm{k}}\right)+\ldots$

By analyzing the waveform frequency spectrum and adjusting the coefficient AK of each harmonic wave (the "taste" of timbre by human ear mainly depends on the proportion of fundamental wave and harmonic wave), the waveform of all kinds of timbres can be produced. Therefore, for the common electronic organ, the timbres are simulated by the attenuation or amplification of certain harmonic wave. This can be realized by RC or LC filter network.

How to make the electronic organ have all kinds of timbres with SCM? Let's take an example of sine wave, as shown in figure 3.

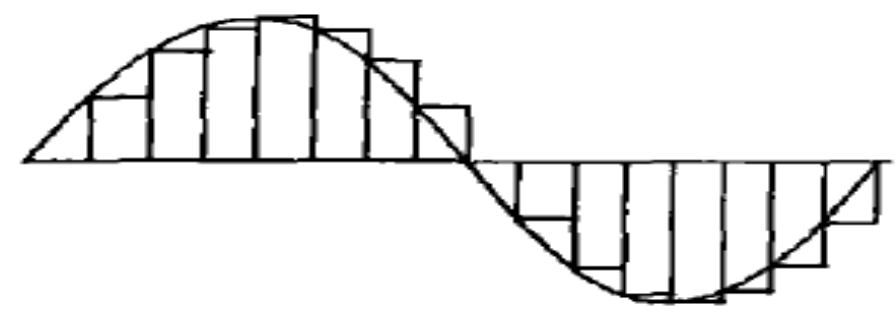

Fig. 3 Analog sine wave

The sine wave can be simulated by a set of sine rectangular waves. Where there are many sines, the simulated sine wave approaches to the original sine wave (which is same with the principle of calculus), which, however, is the simulated waveform. If the width of rectangular wave (actually duration) can be changed, the waveform period or the frequency will be changed as shown in figure 4 . This can be realized by setting the corresponding initial value of timber according to the different keys (corresponding to different pitch).

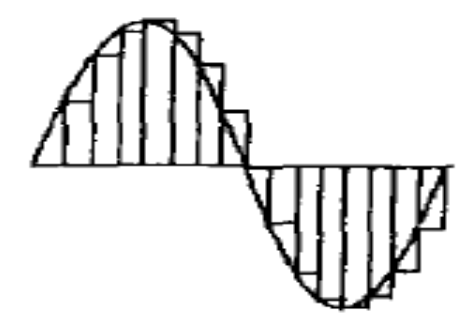

Fig. 4 Change the sine wave frequency

A set of sine waves are converted into digital quantity under the bipolar circumstance. The data below are obtained by 8-bit data representation method with 32 sine waves in table 1 . 
Table. 132 sine waves

\begin{tabular}{|c|c|c|c|c|c|c|c|}
\hline DB 80H & $96 \mathrm{H}$ & $\mathrm{CSH}$ & $\mathrm{AEH}$ & $\mathrm{D} 8 \mathrm{H}$ & $\mathrm{E} 9 \mathrm{H}$ & $\mathrm{FSH}$ & $\mathrm{FDH}$ \\
\hline $\mathrm{FFH}$ & $\mathrm{FDH}$ & $\mathrm{FSH}$ & $\mathrm{E} 9 \mathrm{H}$ & $\mathrm{DBH}$ & $\mathrm{CSH}$ & $\mathrm{AEH}$ & $96 \mathrm{H}$ \\
\hline $80 \mathrm{H}$ & $66 \mathrm{H}$ & $4 \mathrm{EH}$ & $38 \mathrm{H}$ & $25 \mathrm{H}$ & $15 \mathrm{H}$ & $09 \mathrm{H}$ & $04 \mathrm{H}$ \\
\hline $\mathrm{OOH}$ & $04 \mathrm{H}$ & $09 \mathrm{H}$ & $15 \mathrm{H}$ & $25 \mathrm{H}$ & $38 \mathrm{H}$ & $4 \mathrm{EH}$ & $66 \mathrm{H}$ \\
\hline
\end{tabular}

As the width of rectangular waves should be controlled with timer, the initial value of timer corresponding to each pitch should be calculated as shown in table 2 .

Table. 2 Initial value of timber corresponding to each pitch

\begin{tabular}{|c|c|c|c|c|}
\hline The kye number & The pitch & Mhz & TH0 & TL0 \\
\hline 1 & $\mathrm{a}$ & 220 & $\mathrm{FF}$ & 72 \\
\hline 2 & $\mathrm{~b} 1$ & 247 & $\mathrm{FF}$ & 81 \\
\hline 3 & $\mathrm{c} 1$ & 262 & $\mathrm{FF}$ & 89 \\
\hline 4 & $\mathrm{~d} 1$ & 294 & $\mathrm{FF}$ & 96 \\
\hline 5 & $\mathrm{e} 1$ & 330 & $\mathrm{FF}$ & $\mathrm{A} 1$ \\
\hline 6 & $\mathrm{f} 1$ & 349 & $\mathrm{FF}$ & $\mathrm{A} 6$ \\
\hline 7 & $\mathrm{~g} 1$ & 932 & $\mathrm{FF}$ & $\mathrm{B} 0$ \\
\hline 8 & $\mathrm{a} 1$ & 440 & $\mathrm{FF}$ & $\mathrm{B} 9$ \\
\hline 9 & $\mathrm{~b} 1$ & 494 & $\mathrm{FF}$ & $\mathrm{C} 1$ \\
\hline 10 & $\mathrm{c} 2$ & 523 & $\mathrm{FF}$ & $\mathrm{C} 4$ \\
\hline 11 & $\mathrm{~d} 2$ & 587 & FF & CB \\
\hline 12 & $\mathrm{e} 2$ & 659 & FF & D1 \\
\hline
\end{tabular}

Note: The data in the table are obtained based on $12 \mathrm{MHz} 32$ rectangular waves and 16-bit timber; 12 keys are listed in the table because the author only sets four functional keys (with recording function) in the specific realization. However, to highlight the simulated timbre in writing the paper, the recording function is canceled in this paper.

\section{Specific realization steps}

Taking the simulation of sine wave as the example, the device is selected and the circuit is designed according to different function as shown in figure 5 .

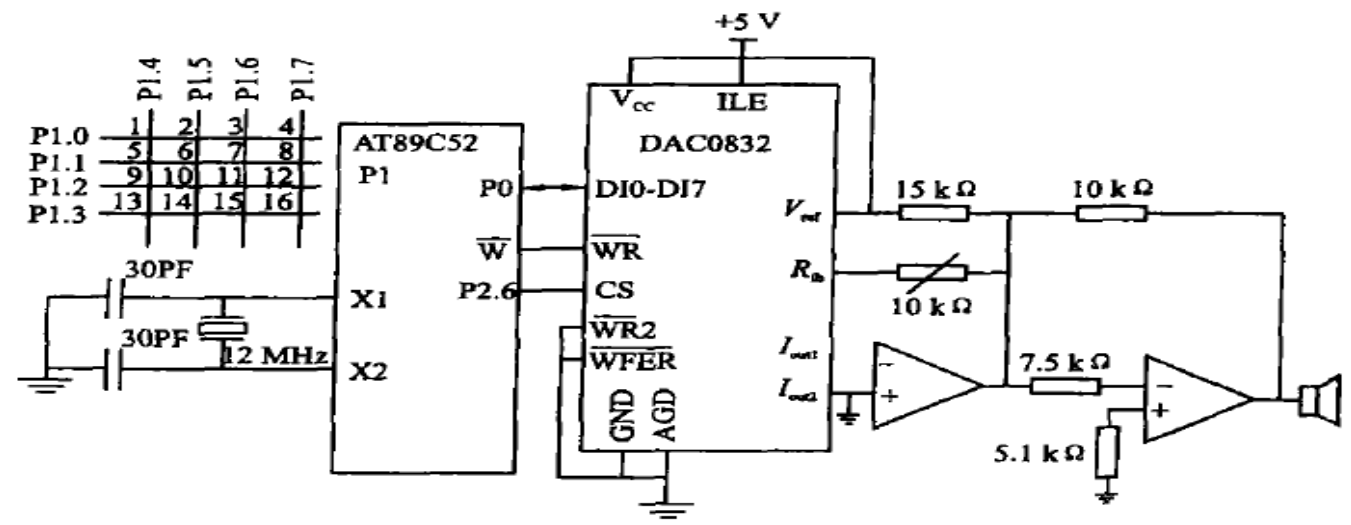

Fig. 5 Analog circuit design of a sine wave

The process flow in playing state is shown in figure 6 . 


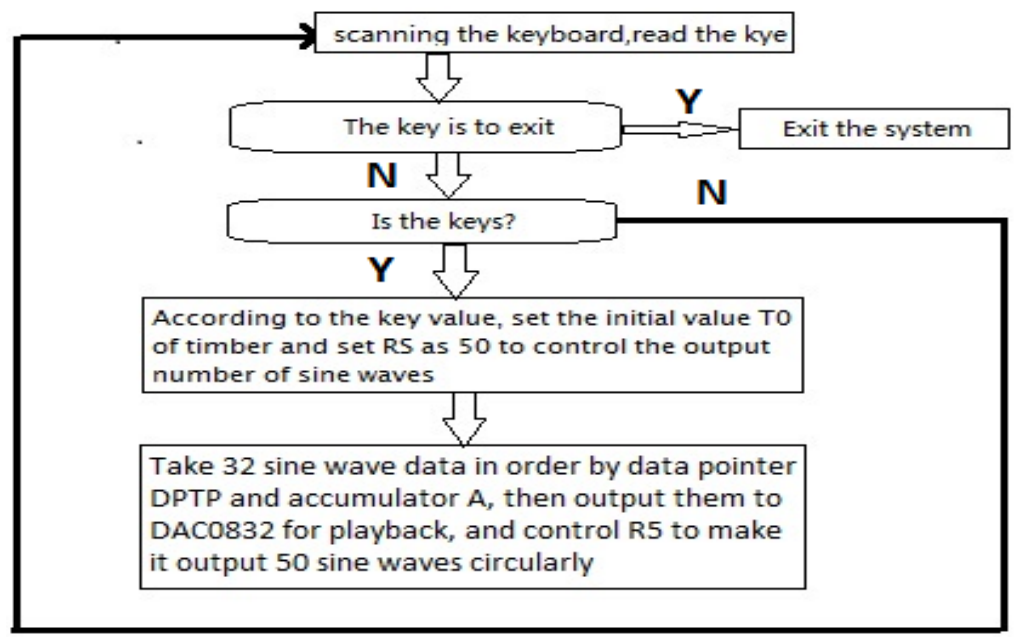

Fig. 6 The process flow in playing state

\section{Conclusion}

For timbre of uncomplicated waveform, the sound effect produced by the method stated in this paper is lifelike. However, if the waveform is complicated, it is required to use high-speed SCM upon design and at the same time, use more waveform data, improve the accuracy and programming method and control the execution time of planning program and data transmission time of SCM and external chip, thus the sound will not be distorted. The simulation of timbre of electronic organ by this method can greatly improve the complexity and polytrope problem of circuit design.

\section{References}

[1] Weng Jianwei, Practical Fundamental Musical Theory, Higher Education Press, Beijing, 2003.

[2] Yu Yongquan, ATMEL89 Series SCM Applied Technology, Beijing University of Aeronautics and Astronautics, Beijing, 2002.

[3] Wang Bing, Design of Micro Electronic Organ with SCM, Electronic Engineer, 2002, p.8-10. 